\title{
Research on the Development Course of Outdoor Sports in China
}

\author{
Chen Bo \\ School of Physical Education of Changzhou University, Changzhou,Jiangsu, China, 213164 \\ Email: cb@cczu.edu.cn.
}

Keywords: Outdoor sports; Development; Course; Football ; Rugby

\begin{abstract}
This paper has researched the development course of outdoor sports in China by the method of literature. The results show that there are three distinct stages in the introduction and popularization of outdoor sports in our country, which is defined mainly according to the outdoor activities' scale, influence and characteristics. In the three stages, the events of outdoor sports are gradually developing from simple to complex and diverse.
\end{abstract}

\section{Introduction}

The UK is known as the birthplace of outdoor sports. The first industrial revolution brought a series of social problems. The national political group began to push sports, like swimming, skating, fishing, high jump, long jump, cricket, golf, hockey, football and rugby. Outdoor sports were regarded as the main part of this trend, which was favored by the masses with its leisure means and free and casual way. Soon, outdoor sports were regarded as a kind of fashion elements, quickly swept across the western world, and began to spread into other countries. A variety of outdoor sports associations were established in succession and quickly spread all over the world. When outdoor sports were first introduced to our country, many people recognized them as maximal sports of "challenging life and nature, and exploring adventurous places"[1-3]. After several years of development, more and more people began to participate in outdoor sports. The influence on society, nature and people became more and more deeply. People began to know more about outdoor sports and changed some of their ideas on outdoor sports. The paper has researched the development course of our outdoor sports and provided certain theoretical basis for the further development of our outdoor sports in the future.

\section{The Development Course of Outdoor Sports in China}

From 1950s to Early 1990s: the Learning and Exploring Stage. The outdoor sports are represented by mountaineering expedition in early stage. The mountaineering team of All China Federation of Trade Unions (ACFTU) was established in 1956. It is our first national mountaineering team. After two years, the State Sports Committee formally established the Mountaineering Department, and founded the Chinese Mountaineering Association in the same year, taking the State Mountaineering Department and the national mountaineering team in. The Mountaineering Association as China's earliest mountaineering organization, has organized several mountain adventure activities, which have a great impact at home and abroad. Among them, the influence of three mountaineering activities at Mount Everest is far-reaching. These activities respectively were Mount Everest Mountaineering from the north slope in 1960, Collective Mount Everest Mountaineering in 1975 and Mount Everest Mountaineering participated by a mountaineering team of Chinese, Japanese and Nepalese jointly in 1988 which achieved a big leap of climbing up from the north and down from the south and climbing up from the south and down form the north. In the meantime[4], other outdoor sports that have been popular in Europe and the United States, like rock climbing, were gradually introduced into China. But due to restrictions in funding, equipment, space, experience and etc., these outdoor sports developed slowly in our country. In view of the large development room, the Chinese Mountaineering 
Association also began to pay attention to its development. In 1897, the Chinese Mountaineering Association sent relevant personnel to Japan to learn about the climbing skills and activity rules. In October of the same year, we held the first rock climbing competition at Natural Rock Climbing Wall of Beijing Huairou Dashuiyu Reservoir. In 1990, we built our first artificial rock climbing field and hosted the related events[5].

From 1990s to About 2003 - the Rapid Rising Stage. "China's large-scale folk outdoor sports began in the period from 1995 to 1996. Originally some advanced universities followed foreign universities and established the mountaineering teams. For example, the Shanying Society of Peking University was founded in 1989. As these students graduated and these sports were spread, the outdoor sports gradually were spread to society, and become fashionable urban exercises", described by the former Secretary General of the Chinese Mountaineering Association Liang Pu on the rise of our outdoor sports[6].

It is for only about 15 year that we have started to develop outdoor sports in a large-scale manner in our country. The most characteristic outdoor adventures and maximal sports have been booming in recent years in our country. From the beginning of 1998, outdoor sports have been quietly rising in Wuhan, Beijing, Shanghai, Guangzhou and other places of China. A variety of media gave strong report, and quickly outdoor sports became a kind of fashion and soon spread to other big cities in China. Through the publicity, report and promotion of media, outdoor clubs developed all over the country like bamboo shoots after a spring rain. In 1990, "Kunming Mountaineering Expedition Association" was founded, and it was the earliest semi-commercial outdoor adventure organization in China. In 1997 the Sanfu Outdoor Sports Club was established, and it was one of the most influential outdoor sports clubs in Beijing. At the same time, non-commercial and commercial outdoor sports forum and the PORTAL appeared in large numbers, providing a convenience and quick platform for the development of outdoor sports, and playing a positive role on the communication, organization and management of outdoor sports and relevant activities[7-8].

From 2004 until Now: the Standardized Development Stage. In April 2005, the State Sports General Administration has officially classified the mountaineering outdoor sports as a conventional event, under the care of the subordinate of the State Sports General Administration - the Mountaineering Management Center, which officially marked that our outdoor sports have entered the conventional development way.

In 2004, the State Ministry of Education established the subject of "outdoor survival training for college students", and planed to introduce the training content into our colleges and universities to develop experimental teaching in the following two years. At present, many universities have opened the outdoor survival quality training courses in our country, and set the outdoor sports, such as field quality development and field survival skill training, as required courses or elective courses. We set up this kind of courses as basic education, which has an important meaning for the healthy, benign and orderly development of outdoor sports. At same time, we have formed an outdoor sports market with outdoor sports group as main body and clubs as carrier, relying on outdoor products stores at present stage. The formation of outdoor sports market has a huge impetus function on the commercial operation, organization, management and supervision of the outdoor sports.

The three development stages of outdoor sports have reflected the developing law of our outdoor sports "introduction - rising - standardization", and the development course of outdoor sports from the national management level into people's life. It is also a reflection of the development of our economy and culture and the improvement of people's living standard. With the function of improving public health, the outdoor sports have a broad mass foundation in our country and are becoming a part of the mass healthy culture. 


\section{The Development Type of Outdoor Sports in China}

Water Sports and Sailing Class. Diving includes underwater swimming, underwater directional sport, underwater shooting and other underwater events. This class of sports requires sportsmen to wear diving suit, carry oxygen bottles and wear diving facial mask and have a respirator in the mouth. The oxygen bottle is connected with the respirator on the back. The participators can only participate this sport after a relatively strict training. This class of sports is for the purpose of physical exercise, leisure and entertainment. It is widely loved by the public, but also there are certain risks.

Sailing includes surfing, water skiing, windsurfing, sampan sailing, boat sailing, yacht, motorboat, water scooter, rafting and other over water events. This class of sports requires to be completed with certain sailing equipment. It is carried out at sea from one land to another land, or in a single route loop. It has higher requirements on participators' steering and operating skills, because there are certain risks. Due to its thrilling, sportful and challenging characteristics, it is highly sought after by some outdoor sports enthusiasts.

Land Sports and Bicycling. Hiking includes walking, march, running, fast walking, orienteering, hunting and other land walking events. It is classified according to travel path and region difference, like suburb, country, mountain, river and etc.. The participators generally wear professional clothing and shoes for a long-distance hiking activity in order to protect themselves. With relative lower risk, it is one of the most popular outdoor sports.

Bicycling includes long-distance road bicycling, cross-country mountain bicycling, bicycle motocross and downhill mountain biking and other land bicycling events. This class of sports requires to be completed with certain equipment. It has a higher requirements on participators' driving skills, because there are certain risks. However, it is relative convenient and popular.

Mountain Sports and Underground Activities. Mountaineering includes mountain hiking, mountain climbing, snow shoeing and other mountain land sports. Broadly speaking, there is no restriction. All people can participate in freely. From a narrow sense, it is particular about equipment and skills. It has high requirements on physical quality. Participators need to overcome a variety of difficulties in order to complete the activity.

Downhill includes skiing, slides, grass skiing, rock down and other land mountain events, which originates from mountain adventure and military raids. Through continuous innovation of sports enthusiasts, it has been added with similar rock climbing, bungee jumping and etc., belonging to maximal sports events. This class of events is very dangerous and challenging.

Climbing includes rock climbing, stone climbing, climbing with equipment and other mountain land events. It has high requirements on participator's physical quality and climbing techniques, because these sports are dangerous and challenging.

Caving includes natural cave, artificial cave, underwater karst cave and other mountain land events. It has high requirements on participators' comprehensive abilities, such as using one rope for lifting, rock climbing, swimming, diving and mastering rescue and first aid techniques. This class of sports is very dangerous and challenging.

Camping and Hunting. Includes outside sleeping and camping, picnic hunting, field training, quality development, net casting, fishing, barbecue cooking, terrain survey, ore collecting, folk-custom investigation, historic site exploration, anecdote interviewing and other land survival skill events. These sports are wide in scope, very sportful and recreational, and have high requirements on physical quality, activity skill and equipment. With universal training and activity significance, these sports are accepted and carried out widely.

Motor Vehicles and Ships and Airsports. Includes cross country on mountain, road race, long-distance travel, car racing, cross country, exploration, travel, vacation, skiing, skating, water skiing, roller skating, skateboarding, bungee jumping, rock hopping, skydiving, paragliding, powered parachute, hot air balloon, glider, ultralight and other land maximal and sub-maximal sports. This class 
of events is wide in scope, with high risks and has high requirement on physical quality, driving and operating skills and activity equipment. It is the best choice of nature lovers and outdoor sports challengers.

Entertainment and Military Activities. Includes grass game, ball, basketball, volleyball, football, badminton, tennis, sandbags, sledge, semaphore communication, bicycle, telegram and telephone communication, monocycle, light communication, air gun, dart crossbow, archery, paintball field and etc., generally covering mountain, land, water and air events and integrating entertainment, summer camp and quality development. These sports are very sportful and recreational, less dangerous, so they are the best choice of vast teenagers.

\section{Conclusions and Suggestions}

Conclusion. The three development stages of our outdoor sports have their own characteristics, which are mainly reflected in the classification of outdoor sports, organization and participation units and the popularization level.

(1) In the first stage, the first outdoor sport we carried out was mountaineering at beginning. Soon afterwards, we introduced some European and American mainstream outdoor sports, mainly including rock climbing, hiking and bicycling. The main organizers were the Chinese Mountaineering Association and its subordinate units. The outdoor sports participators mainly were national professional sportsmen. With single universal level, the outdoor sports were not promoted on a national scale[9].

(2) In the second stage, our outdoor sports were changing from original mountaineering, rock climbing, hiking and cycling and other land and mountain events to land, underground and water sports. It is worth mentioning that some high-challenging and advanced European and American outdoor sports also began to appear in China during this period. There were diversified outdoor sports organizers and undertakers, covering national-level units, provincial-level units and municipal units. The commercial and non-commercial folk spontaneous clubs appeared and they were important carriers to organize and manage outdoor sports. The participators were no longer only about national professional sportsmen but also the public. The purpose of sports was altered from simple competition to leisure, relaxation, fitness, competition and etc.

(3) In the third stage, the outdoor sports we carry out basically involve in all events that our natural environment conditions can bear. Guided by the government, we introduce equipment and technology required for these events. By making relevant policies and regulations, we set up training pilot sites in physical education colleges and universities, and encourage the development of private commercial and non-commercial institutions. The activities we carry out are basically synchronous with international level. With the comprehensive encouragement and mobilization of national fitness, the masses could participate all outdoor sports through the government and commercial organizations. The outdoor sports have become an important part of people's leisure, entertainment and health life. Due to the change of development range and intensity, some safety accidents occur frequently, which reflect some ethical absence in outdoor sports industry[10].

Suggestions. More and more people start to participate in outdoor sports. In order to meet the expanding market demand, we are making the sales market of outdoor sports products an industrial scale. In the future, we may gradually get rid of gyms to carry out outdoor sports. We can walk into nature, indulge in the mountains and seek the nature and significance of human survival and living in nature. By perfecting the ethical principle and norms of outdoor sports constantly, we can establish the relationship between outdoor sports and people, people and nature, people and society. However, there is still a certain distance between our outdoor sports and international outdoor sports. On one aspect, we are in the primary stage of socialism with relative not high economic development level. On the other hand, the outdoor sports are not developed completely and intensively in our country. Our 
operation concept and management idea are not very advanced and innovative, so our development potential of outdoor sports has not been dug out really.

\section{References}

[1] Li Shuping, Zou Kai. Risk Management on Outdoor Sports. Guangzhou: Guangdong Science and Technology Press, 2008.

[2] Meng Gang. Outdoor Sports. Beijing: Beijing Normal University Press, 2010.

[3] Li Liyu. Outdoor Sports and Outdoor Training. Hangzhou: Zhejiang University Press, 2012.

[4] Tao Qian, Liang Haifei. Sports Ethics and Harmonious Society. Journal of Sports Culture, 2005 (10).

[5]Li Wanxin. Investigation and Analysis on the Physical Exercises Condition of Nonprofessional Physical Education Students at Common Universities [J]. Western Education, 2013 (30).

[6]Liu Weifeng, Tian Jian. Investigation \& Counter-measure Research on the Physical Exercises Condition of University Graduate Students [J]. Sichuan Sports Science, 2005(3).

[7]Sun Liang. Research on Extracurricular Physical Exercises of University Students [J]. Journal of Yanbian Institute of Education, 2010(1).

[8]Dai Xingxing, Wang Bin. Research on Extracurricular Physical Exercises of University Students in Henan Province [J]. Science \& Technology Information, 2010(31).

[9] Wang Li. Sociological Thinking on Outdoor Sports. China After-school Education, 2009 (12): 321.

[10] Pan Jingwu. Sports Ethics. Beijing: Beijing Institute of Physical Education, 1987. 